\title{
Work-Related Musculoskeletal Disorders Among Medical Practitioners in the Hospitals of Al'Qassim Region, Saudi Arabia
}

Yasser Alwabli ${ }^{1}$, Moath A. Almatroudi ${ }^{2}$, Meshari A. Alharbi ${ }^{2}$, Muath Y. Alharbi ${ }^{2}$, Sultan Alreshood ${ }^{2}$, Faisal A. Althwiny ${ }^{2}$

1. Orthopaedics, College of Medicine, Qassim University, Buraidah, SAU 2. Orthopaedics, Unaizah College of Medicine, Qassim University, Unaizah, SAU

Corresponding author: Faisal A. Althwiny, f.a.thwiny@gmail.com

\section{Abstract \\ Introduction}

Work-related musculoskeletal disorders (WMSDs) have a significant impact on the workplace; they have been growing as a problem in our population, especially among healthcare practitioners. The aim of the study was to determine the prevalence of the condition, evaluate the WMSDs experienced by practitioners in different specialties in different hospitals in the Al'Qassim region, and study various risk factors that contribute to the development of WMSDs.

\section{Methods}

After gaining the Institutional Review Board (IRB) approval, a cross-sectional study was conducted among all medical care practitioners at hospitals in the Al'Qassim region. Data were collected using a validated, standardized, and self-administered questionnaire. The data were analyzed using the SAS software version 9.4 (SAS Institute Inc., Cary, NC).

\section{Results}

The study result revealed that out of 382 participants, just over half (209, 54.7\%) experienced WMSDs. Among them, 103 (27.54\%) were males, and 271 (72.54\%) were females. The average age of participants was $31.25 \pm 6.82$ years. Participants with experience of 6-10 years were twice as likely to develop WMSDs compared to participants with fewer years of experience [odds ratio (OR): 2.342 ; $95 \%$ confidence interval (CI): 1.062-5.168; $\mathrm{p}=0.0350]$. There was no significant difference in terms of past medical history between the two groups except for the history of having low back pain, which was more common in participants with WMSDs (77.59\% versus $22.41 \%$; $\mathrm{p}:<0.0001)$, and neck pain $(74.19 \%$ versus $25.81 \%$; $\mathrm{p}=$ $0.0003)$. Almost all job risk factors varied significantly between the groups (p: $<0.05)$. Prevalent risk factors included performing the same task over and over again $(134,37.96 \%)$, treating an excessive number of patients in one day $(127,35.88 \%)$, and working in the same positions $(126,35.8 \%)$.

Received 05/03/2020 Review began 05/09/2020 Review ended 05/20/2020 Published 05/31/2020

\section{(c) Copyright 2020} Alwabli et al. This is an open access article distributed under the terms of the Creative Commons Attribution License CC-BY 4.0., which permits unrestricted use, distribution, and reproduction in any medium, provided the original author and source are credited.

\section{Conclusions}

Based on this study findings, we can conclude that WMSDs affect a high proportion of healthcare professionals; the female gender and more than six years of experience were found to be major predictors for WMSDs. Pain in the lower back, shoulders, and neck were the most frequently reported musculoskeletal complaints (MSCs). Hence, we recommend the incorporation of musculoskeletal disorder prevention programs in the hospitals' educational programs as they will reduce the rate of WMSDs and ensure the health and well-being of healthcare practitioners.

Categories: Physical Medicine \& Rehabilitation, General Surgery, Orthopedics

Keywords: musculoskeletal, wmsd, healthcare practitioners, risk factor

\section{Introduction}

Musculoskeletal complaints (MSCs) in the workplace have a significant impact on the health of healthcare practitioners. They have been growing as a problem in the population, especially among healthcare workers, and they are an important cause of temporary work disability [1-3]. MSCs include a wide variety of degenerative and inflammatory disorders that can affect the joints, muscles, tendons, ligaments, supporting blood vessels or even peripheral nerves, and susceptible body parts such as lower back, neck, shoulders, arms, forearms, hands, and lower extremities [4]. The MSCs have a significant negative impact on healthcare practitioners during their duty. Physicians, nurses, physiotherapists, and other healthcare practitioners face repetitive trauma and constant strains in their routine patient care activities that cause chronic illnesses and musculoskeletal injuries [5]. Work-related musculoskeletal disorders (WMSDs) are defined as musculoskeletal disorders that result from an event that is related to work [6]. Working as a healthcare provider is identified as an important risk factor for WMSDs [3,7-9]. Healthcare workers have a higher 
prevalence of low back pain compared to other hospital and industrial workers [10]. A study about WMSDs that was done in Saudi Arabia among dental professionals showed that 85\% experienced MSCs in some form [11]. Another study conducted on WMSDs among healthcare practitioners in India showed that $50.7 \%$ of the participants experienced MSCs [3]. Also, a study in China showed that 70\% of nurses experienced MSCs [12]. Back, neck, knee, and shoulder complaints are most commonly seen among healthcare practitioners [3,1315]. The aim of the study was to determine the prevalence of this condition, evaluate the WMSDs experienced by practitioners in different specialties in different hospitals in the Al'Qassim region, and study the different risk factors that contribute to the development of WMSDs.

\section{Materials And Methods}

After gaining the institutional Review Board (IRB) approval, a cross-sectional study was conducted among the medical practitioners in the Al'Qassim province in 2019. The study was carried out at the following three governmental hospitals in the Al'Qassim province: King Saud Hospital, Unaizah; Buraidah Central Hospital, Buraidah; and Ar Rass General Hospital, Ar Rass. The hospitals were chosen by a simple random technique. The sample size was calculated by using OpenEpi software version 3 , and the result was as follows: $\mathrm{n}=\left[\mathrm{DEFF}^{*} \mathrm{~Np}(1-\mathrm{p})\right] /\left[\left(\mathrm{d} 2 / \mathrm{Z} 21-\alpha / 2 *(\mathrm{~N}-1)+\mathrm{p}^{*}(1-\mathrm{p})\right]=375\right.$. All medical care practitioners at Al'Qassim hospitals, including physicians, nurses, lab workers, and other allied medical practitioners, were included in the study. All hospital administration staff were excluded.

Data were collected using a validated pretested questionnaire used in the study conducted by Yashwant $S$ et al. 2014 [3]. The study tool validation was approved by experts and by the Alpha-Cronbach test.

The questionnaire was in English and consisted of different standardized questionnaires. The first part of the questionnaire consisted of demographic data, lifestyle, and occupational history. The second part comprised the Self-reported Ergonomic Hazards at Workstation Questionnaire. The third part consisted of the Job Factors Questionnaire, which was administered using an ordinal scale of 0-10 to determine if the symptoms were work-related. Finally, the last part comprised the Nordic Musculoskeletal Questionnaire (NMQ) to identify the presence of self-reported musculoskeletal symptoms in the preceding 12 months (Table 6, Appendix).

A pilot study was done on $10 \%$ of our sample size (38 participants; 19 male and 19 female) before starting our actual study in order to test the validity of our questionnaire and to estimate the timing needed for each participant to complete the questionnaire. Specific data were collected as identified in the questionnaire papers and were listed on Excel spreadsheets (Microsoft, Redmond, WA). The data were analyzed using the SAS system version 9.4. (SAS Institute Inc., Cary, NC). Questionnaire scores were calculated according to the definitions described in the questionnaire. For categorical variables, frequencies and percentages were calculated, whereas, for continuous variables, results were presented as mean ${ }^{ \pm}$standard deviation (SD), or median \pm interquartile range (IQR) in case of skewed data. Association between categorical variables was analyzed using the chi-squared test $(\chi 2)$, whereas in cases of zero or small cells, Fisher's exact test was implemented instead. A two-sample t-test was used for the normally distributed continuous variables; otherwise, a Mann-Whitney (Wilcoxon) two-sample test was implemented. Multivariable logistic regression analysis was performed. Odds ratios (ORs) with 95\% confidence intervals (CIs) were expressed relative to a reference baseline category. A p-value of less than 0.05 was considered statistically significant. All results were summarized in tables and figures.

\section{Results}

A total of 382 participants were included based on the inclusion and exclusion criteria; 103 (27.54\%) were males, 271 (72.54\%) were females; the gender of eight participants was not provided in their answers to the questionnaire. The average age of the participant was $31.25 \pm 6.82$ years. Among the participants, 209 (54.7\%) had experienced WMSDs. The age, gender, and other participant demographics are presented in Table 1 .

\begin{tabular}{|c|c|c|c|c|c|c|c|}
\hline Demographics & $\begin{array}{l}{ }^{*} \text { Total } \\
\text { participants } \\
(n=382)\end{array}$ & $\begin{array}{l}\text { Participants } \\
\text { with WMSDs } \\
(\mathrm{n}=209)\end{array}$ & $\begin{array}{l}\text { Participants } \\
\text { without } \\
\text { WMSDs } \\
(n=173)\end{array}$ & $\begin{array}{l}\mathrm{P} \text { - } \\
\text { value }\end{array}$ & $\begin{array}{l}\text { Frequency of missing } \\
\text { data in demographic } \\
\text { categories ( } n)\end{array}$ & $\begin{array}{l}\text { Frequency of missing data } \\
\text { in participants with and } \\
\text { without WMSDs (n) }\end{array}$ & $\begin{array}{l}\text { Total } \\
\text { frequency of } \\
\text { missing data } \\
\text { (n) }\end{array}$ \\
\hline $\begin{array}{l}{ }^{a} \text { Age, median } \\
(\text { IQR })^{\mathrm{a}}\end{array}$ & 29.00 (8) & $29.00(8)$ & $30.00(9)$ & 0.2806 & - & - & - \\
\hline $\begin{array}{l}{ }^{\mathrm{a} B M} \text {, median } \\
\text { (IQR) }\end{array}$ & 24.00 (5.54) & $24.32(6)$ & $24.57(5.25)$ & 0.6606 & - & - & - \\
\hline $\begin{array}{l}\text { aNumber of } \\
\text { shifts, median } \\
\text { (IQR) }\end{array}$ & $8.00(10)$ & 3.00 (1) & $3.00(0)$ & 0.6415 & - & - & - \\
\hline & & & & & & & \\
\hline
\end{tabular}




\section{Cureus}

\begin{tabular}{|c|c|c|c|c|c|c|c|}
\hline $\begin{array}{l}\text { shift, median } \\
\text { (IQR) }\end{array}$ & $8.00(5.5)$ & $8.00(1)$ & $8.00(0)$ & 0.0135 & - & - & - \\
\hline Sex & & & & 0.2584 & 8 & & \\
\hline Male, n (\%) & $\begin{array}{l}103 \\
(27.54 \%)\end{array}$ & $52(50.98 \%)$ & $50(49.02 \%)$ & & - & 1 & 9 \\
\hline Female, n (\%) & $\begin{array}{l}271 \\
(72.46 \%)\end{array}$ & $153(57.52 \%)$ & $113(42.48 \%)$ & & - & 5 & 13 \\
\hline Marital status & & & & 0.0876 & 12 & & \\
\hline Married, n (\%) & $\begin{array}{l}209 \\
(56.49 \%)\end{array}$ & 108 (52.17\%) & $99(47.83 \%)$ & & - & 2 & 14 \\
\hline $\begin{array}{l}\text { Unmarried, } \mathrm{n} \\
(\%)\end{array}$ & $\begin{array}{l}161 \\
(43.51 \%)\end{array}$ & 96 (61.15\%) & $61(38.85 \%)$ & & - & 4 & 16 \\
\hline $\begin{array}{l}\text { Current } \\
\text { occupation }\end{array}$ & & & & & 50 & & \\
\hline Physician, n (\%) & $41(12.42 \%)$ & $23(56.1 \%)$ & $18(43.9 \%)$ & 0.8753 & & 0 & 50 \\
\hline Nurse, n (\%) & $\begin{array}{l}215 \\
(64.95 \%)\end{array}$ & $123(58.3 \%)$ & 88 (41.7\%) & 0.6449 & - & 4 & 54 \\
\hline $\begin{array}{l}\text { Physiotherapist, } \\
\text { n (\%) }\end{array}$ & 19 (5.76\%) & $13(68.42 \%)$ & $6(31.58 \%)$ & 0.3096 & - & 0 & 50 \\
\hline $\begin{array}{l}\text { badiologist, n } \\
\text { (\%) }\end{array}$ & $9(2.72 \%)$ & 4 (44.44\%) & 5 (55.56\%) & 0.5038 & - & 0 & 50 \\
\hline $\begin{array}{l}\text { Lab technician, } \\
\text { n (\%) }\end{array}$ & $15(4.55 \%)$ & $7(46.67 \%)$ & 8 (53.33\%) & 0.3971 & - & 0 & 50 \\
\hline $\begin{array}{l}\text { Pharmacist, } \mathrm{n} \\
(\%)\end{array}$ & $23(6.97 \%)$ & $15(65.22 \%)$ & $8(34.78 \%)$ & 0.4219 & - & 0 & 50 \\
\hline $\begin{array}{l}\text { bOther } \\
\text { occupations, n } \\
\text { (\%) }\end{array}$ & 10 (3.03\%) & 3 (33.33\%) & $6(66.67 \%)$ & 0.1787 & - & 1 & 51 \\
\hline
\end{tabular}

\section{TABLE 1: Comparison of demographic factors between participants with WMSDs and without WMSDs}

WMSDs: work-related musculoskeletal disorders; IQR: interquartile range

* If the numbers do not add up to the total, it represents missing data, and the shown \% figure represents a valid percentage

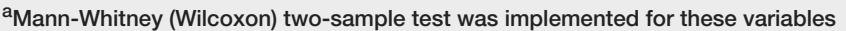

${ }^{\mathrm{b}}$ Fisher's exact test was implemented for these variables

There was no significant difference between groups in terms of past medical history except for the history of having low back pain, which was more common in participants with WMSDs (77.59\% versus $22.41 \%$; p: $<.0001)$, and neck pain $(74.19 \%$ versus $25.81 \% ; p=0.0003)$. In this study population, $30.7 \%$ of the participants were involved in physical activities such as sports and exercises routinely. The overall number of smokers was 26 (6.86\%), of which 14 participants experienced WMSDs, and 12 did not.

Higher rates of almost all the self-reported ergonomic hazards at workstation were seen in participants with neck flexion of more than 20 degrees among WMSD participants $(169,64.50 \%)$ compared to those who did not have WMSDs $(93,35.5 \%)(\mathrm{p}=0.0001)$ (Table 2$)$. 


\section{Cureus}

\begin{tabular}{|c|c|c|c|c|c|c|c|}
\hline $\begin{array}{l}\text { Workstation } \\
\text { hazards }\end{array}$ & $\begin{array}{l}{ }^{*} \text { Total } \\
\text { participants } \\
(\mathrm{n}=382), \mathrm{n} \\
(\%)\end{array}$ & $\begin{array}{l}\text { Participants } \\
\text { with WMSDs } \\
(n=209), n(\%)\end{array}$ & $\begin{array}{l}\text { Participants } \\
\text { without } \\
\text { WMSDs } \\
(n=173), n(\%)\end{array}$ & $\begin{array}{l}\text { P- } \\
\text { value }\end{array}$ & $\begin{array}{l}\text { Frequency of missing } \\
\text { data in workstation } \\
\text { hazards categories (n) }\end{array}$ & $\begin{array}{l}\text { Frequency of missing } \\
\text { data in participants with } \\
\text { and without WMSDs (n) }\end{array}$ & $\begin{array}{l}\text { Total } \\
\text { frequency of } \\
\text { missing } \\
\text { data }(n)\end{array}$ \\
\hline $\begin{array}{l}\text { Neck flexion of } \\
\text { more than } 20 \\
\text { degrees }\end{array}$ & $\begin{array}{l}263 \\
(71.27 \%)\end{array}$ & $169(64.50 \%)$ & 93 (35.5\%) & 0.0001 & 13 & 4 & 17 \\
\hline $\begin{array}{l}\text { Arm level higher } \\
\text { than shoulder }\end{array}$ & $\begin{array}{l}190 \\
(51.63 \%)\end{array}$ & 119 (63.64\%) & 68 (36.36\%) & 0.0053 & 14 & 4 & 18 \\
\hline $\begin{array}{l}\text { Repetitive work } \\
\text { of more than } 4 \\
\text { minutes }\end{array}$ & $\begin{array}{l}270 \\
(73.77 \%)\end{array}$ & $164(61.42 \%)$ & 103 (38.58\%) & 0.0020 & 16 & 4 & 20 \\
\hline Forceful work & $\begin{array}{l}210 \\
(56.91 \%)\end{array}$ & 136 (65.70\%) & $71(34.30 \%)$ & 0.0001 & 13 & 4 & 17 \\
\hline $\begin{array}{l}\text { Forward } \\
\text { bending of the } \\
\text { trunk }\end{array}$ & $\begin{array}{l}254 \\
(69.02 \%)\end{array}$ & 162 (64.54\%) & 89 (35.46\%) & 0.0001 & 14 & 4 & 18 \\
\hline $\begin{array}{l}\text { Lateral bending } \\
\text { or twisting of } \\
\text { the trunk }\end{array}$ & $\begin{array}{l}196 \\
(53.41 \%)\end{array}$ & $127(65.80 \%)$ & 66 (34.20\%) & 0.0001 & 15 & 4 & 19 \\
\hline $\begin{array}{l}\text { Prolonged } \\
\text { sitting of more } \\
\text { than } 20 \text { minutes }\end{array}$ & $\begin{array}{l}190 \\
(51.63 \%)\end{array}$ & $123(65.08 \%)$ & $66(34.92 \%)$ & 0.0005 & 14 & 4 & 18 \\
\hline $\begin{array}{l}\text { Prolonged } \\
\text { standing of } \\
\text { more than } 20 \\
\text { minutes }\end{array}$ & $\begin{array}{l}292 \\
(79.78 \%)\end{array}$ & $175(60.76 \%)$ & 113 (39.24\%) & 0.0017 & 16 & 4 & 20 \\
\hline $\begin{array}{l}\text { Lifting, pulling } \\
\text { or pushing }\end{array}$ & $\begin{array}{l}251 \\
(68.02 \%)\end{array}$ & 146 (58.87\%) & $102(41.13 \%)$ & 1724 & 13 & 4 & 17 \\
\hline
\end{tabular}

\section{TABLE 2: Self-reported ergonomic hazards at workstation}

WMSDs: work-related musculoskeletal disorders

"If the numbers do not add up to the total, it represents missing data, and the shown \% figure represents a valid percentage

Job risk factors for different categories are shown in Table 3. Almost all job risk factors significantly varied among the different groups ( $\mathrm{p}:<0.05)$. Performing the same task over and over again (134 participants,

$37.96 \%$ ), treating an excessive number of patients in one day (127 participants, 35.88\%), and working in the same positions (126 participants, 35.8\%) were the top three job risk factors recorded.

\begin{tabular}{|c|c|c|c|c|c|c|c|}
\hline Job risk factor & $\begin{array}{l}\text { Total } \\
\text { participants } \\
\text { (n=382), n } \\
(\%)\end{array}$ & $\begin{array}{l}\text { with WMSDs } \\
(\mathrm{n}=209), \mathrm{n} \\
(\%)\end{array}$ & $\begin{array}{l}\text { without } \\
\text { WMSDs } \\
\text { (n=173), n (\%) }\end{array}$ & P-value & $\begin{array}{l}\text { missing data in } \\
\text { job risk factor } \\
\text { categories (n) }\end{array}$ & $\begin{array}{l}\text { Frequency of } \\
\text { missing data in } \\
\text { participants with } \\
\text { and without WMSDs } \\
\text { (n) }\end{array}$ & $\begin{array}{l}\text { frequency of } \\
\text { missing } \\
\text { data }(n)\end{array}$ \\
\hline $\begin{array}{l}\text { Performing the same task } \\
\text { over and over again }\end{array}$ & & & & $<0.0001$ & 29 & 4 & 33 \\
\hline No problem & $50(14.16 \%)$ & $10(20 \%)$ & $40(80 \%)$ & & & & \\
\hline Minimal to moderate & $\begin{array}{l}169 \\
(47.88 \%)\end{array}$ & $95(5 / .23 \%)$ & $71(42 . / / \%)$ & & & & \\
\hline Major problem & $\begin{array}{l}134 \\
(37.96 \%)\end{array}$ & $93(69.92 \%)$ & $40(30.08 \%)$ & & & & \\
\hline
\end{tabular}




\section{Cureus}

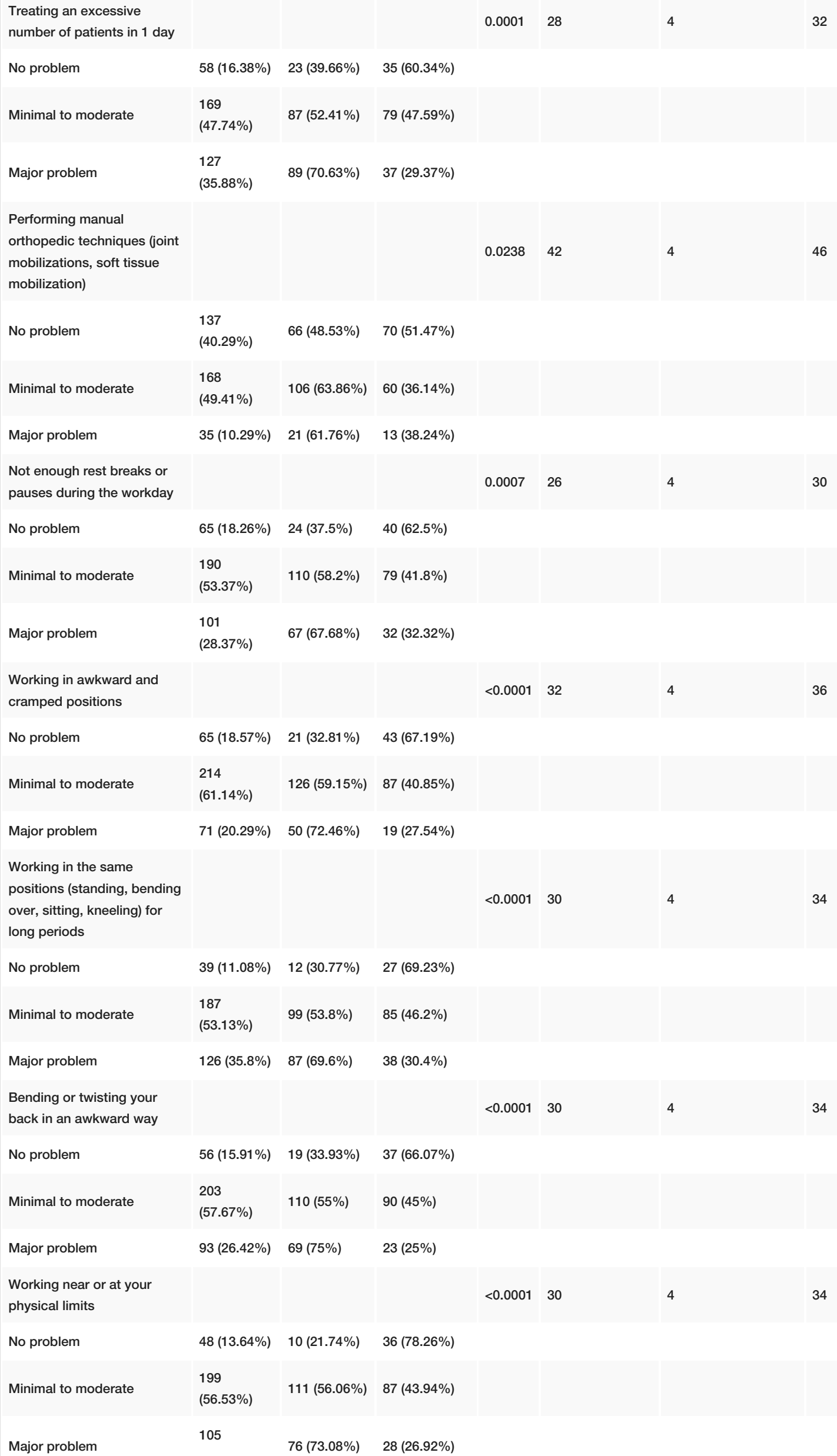




\section{Cureus}

$(29.83 \%)$

Reaching or working away

from your body

$0.0003 \quad 33$

37

No problem

$74(21.20 \%) \quad 27(37 \%) \quad 46(63 \%)$

Minimal to moderate

199

$(57.02 \%) \quad 118(59.9 \%) \quad 79(40.1 \%)$

Major problem

$76(21.78 \%) \quad 51(68 \%) \quad 24(32 \%)$

Continuing to work while

injured or hurt

$0.0259 \quad 31$

No problem

111

(31.62\%)

$50(45.87 \%) \quad 59(54.13 \%)$

Minimal to moderate

(45.58\%)

$97(61 \%) \quad 62(39 \%)$

Major problem

$80(22.79 \%)$

$49(62.03 \%) \quad 30(37.97 \%)$

Lifting or transferring

dependent patients

No problem

$88(25.36 \%) \quad 39(44.3 \%) \quad 49(55.7 \%)$

Minimal to moderate

147

$(42.36 \%) \quad 84(57.93 \%) \quad 61(42.07 \%)$

Major problem

112

(32.28\%)

$69(62.73 \%) \quad 41(37.27 \%)$

Working with confused or

agitated patients

No problem

$92(26.59 \%) \quad 47(52.22 \%) \quad 43(47.78 \%)$

Minimal to moderate

154

$(44.51 \%) \quad 82(53.25 \%) \quad 72(46.75 \%)$

Major problem

100

(28.90\%)

$66(67.35 \%) \quad 32(32.65 \%)$

Carrying, lifting, or moving

heavy materials

No problem

$83(24.27 \%) \quad 40(49.38 \%) \quad 41(50.62 \%)$

Minimal to moderate

167

(48.83\%)

$89(53.3 \%) \quad 78(46.71 \%)$

Major problem

$92(26.90 \%)$

$63(70 \%)$

$27(30 \%)$

Unanticipated sudden

movement or fall by patients

No problem

134

(39.07\%)

$64(48.85 \%) \quad 67(51.15 \%)$

141

Minimal to moderate

$(41.11 \%)$

$88(62.41 \%) \quad 53(37.59 \%)$

Major problem

$68(19.83 \%) \quad 39(58.21 \%) \quad 28(41.79 \%)$

Assisting patients during gait

activities

$0.1391 \quad 39$

4

43

No problem

$95(27.70 \%) \quad 45(48.39 \%) \quad 48(51.61 \%)$

Minimal to moderate

170

(49.56\%)

$98(57.65 \%) \quad 72(42.35 \%)$

Major problem

$78(22.74 \%) \quad 48(63.16 \%) \quad 28(36.84 \%)$

Work scheduling (overtime,

$0.0285 \quad 35$

39

$0.0497 \quad 36$

$0.0109 \quad 40$

44

$0.0746 \quad 39$

43 


\section{Cureus}

\begin{tabular}{|c|c|c|c|c|c|c|c|}
\hline $\begin{array}{l}\text { irregular shifts, longer length } \\
\text { of the workday) }\end{array}$ & & & & $<0.0001$ & 32 & 4 & 36 \\
\hline No problem & $77(22 \%)$ & 27 (36\%) & $48(64 \%)$ & & & & \\
\hline Minimal to moderate & $182(52 \%)$ & 105 (57.69\%) & 77 (42.31\%) & & & & \\
\hline Major problem & $91(26 \%)$ & $66(74.16 \%)$ & $23(25.84 \%)$ & & & & \\
\hline $\begin{array}{l}\text { Inadequate training on injury } \\
\text { prevention }\end{array}$ & & & & 0.0007 & 36 & 4 & 40 \\
\hline No problem & $\begin{array}{l}126 \\
(36.42 \%)\end{array}$ & $54(43.55 \%)$ & $70(56.45 \%)$ & & & & \\
\hline Minimal to moderate & $\begin{array}{l}165 \\
(47.69 \%)\end{array}$ & $105(64.02 \%)$ & $59(35.98 \%)$ & & & & \\
\hline Major problem & 55 (15.9\%) & $36(66.67 \%)$ & $18(33.33 \%)$ & & & & \\
\hline
\end{tabular}

\section{TABLE 3: Self-reported job risk factors among all participants}

WMSDs: work-related musculoskeletal disorders

*If the numbers do not add up to the total, it represents missing data, and the shown \% figure represents a valid percentage

Participants with experience of 6-10 years with an average age of $32.63 \pm 4.53$ years are twice as likely as participants with fewer years of experience and an average age of $25.54 \pm 3.51$ years to develop WMSDs (OR: 2.342; 95\% CI: $1.062-5.168, \mathrm{p}=0.0350$ ). Table 4 shows the multivariate analysis involving female gender, age, body mass index (BMI), experience, and exercise.

\begin{tabular}{|c|c|c|c|c|}
\hline Predictor & Category & Odds ratio & $95 \% \mathrm{Cl}$ for OR & P-value \\
\hline \multirow{2}{*}{ Age } & $30-50$ vs $>50$ & 0.872 & $0.102-7.466$ & 0.9008 \\
\hline & $<30$ vs $>50$ & 0.746 & $0.075-7.453$ & 0.8031 \\
\hline \multirow{3}{*}{ BMI } & Underweight vs obesity & 0.826 & $0.318-2.146$ & 0.6952 \\
\hline & Normal weight vs obesity & 1.578 & $0.704-3.537$ & 0.2684 \\
\hline & Pre-obesity vs obesity & 0.743 & $0.280-1.976$ & 0.5523 \\
\hline Gender & Female vs male & 1.173 & $0.331-4.162$ & 0.8047 \\
\hline \multirow{3}{*}{ Experience } & Experience of $<1$ year & 2.415 & $0.754-7.734$ & 0.1376 \\
\hline & Experience of $1-5$ years & 1.930 & $0.797-4.672$ & 0.1448 \\
\hline & Experience of $6-10$ years & 2.342 & 1.062-5.168 & 0.0350 \\
\hline Exercise & Yes vs no & 0.864 & $0.506-1.476$ & 0.5936 \\
\hline
\end{tabular}

\section{TABLE 4: Predictors of WMSDs among healthcare professionals}

WMSDs: work-related musculoskeletal disorders; BMI: body mass index; CI: confidence interval; OR: odds ratio

The results of the Nordic Musculoskeletal Questionnaire (NMQ), which showed the self-reported symptoms in all the 382 participants, indicated that more than half of the participants $(56.66 \%$ ) had experienced low back pain in the past 12 months, followed by pain in the neck (46.05\%), and shoulders (38.81\%); for many of them, the WMSDs negatively impacted their performance, leading them to seek help (Table 5). 


\begin{tabular}{|c|c|c|c|c|c|c|c|c|c|c|c|c|}
\hline \multirow[t]{2}{*}{ Body part } & \multicolumn{3}{|c|}{$\begin{array}{l}\text { Occurrence of MS problems } \\
\text { during the last } 12 \text { months, } n(\%)\end{array}$} & \multicolumn{3}{|c|}{$\begin{array}{l}\text { Normal activity prevented by } \\
\text { your problems, } \mathrm{n}(\%)\end{array}$} & \multicolumn{3}{|c|}{$\begin{array}{l}{ }^{*} \text { Seen a physician during the last } \\
12 \text { months for this condition, } n \\
\text { (\%) }\end{array}$} & \multicolumn{3}{|c|}{$\begin{array}{l}\text { Occurrence of MS problem } \\
\text { during the last } 7 \text { days, } n \text { (\%) }\end{array}$} \\
\hline & Yes & No & $\begin{array}{l}\text { Total } \\
\text { frequency of } \\
\text { missing } \\
\text { data }(n)\end{array}$ & Yes & No & $\begin{array}{l}\text { Total } \\
\text { frequency of } \\
\text { missing } \\
\text { data }(n)\end{array}$ & Yes & No & $\begin{array}{l}\text { Total } \\
\text { frequency of } \\
\text { missing } \\
\text { data }(n)\end{array}$ & Yes & No & $\begin{array}{l}\text { Total } \\
\text { frequency or } \\
\text { missing } \\
\text { data }(n)\end{array}$ \\
\hline Neck & $\begin{array}{l}163 \\
(46.05 \%)\end{array}$ & $\begin{array}{l}191 \\
(53.95 \%)\end{array}$ & 28 & $\begin{array}{l}62 \\
(18.08 \%)\end{array}$ & $\begin{array}{l}281 \\
(81.92 \%)\end{array}$ & 39 & $\begin{array}{l}42 \\
(12.24 \%)\end{array}$ & $\begin{array}{l}301 \\
(87.76 \%)\end{array}$ & 39 & $\begin{array}{l}82 \\
(24.26 \%)\end{array}$ & $\begin{array}{l}256 \\
(75.74 \%)\end{array}$ & 44 \\
\hline Shoulders & $\begin{array}{l}137 \\
(38.81 \%)\end{array}$ & $\begin{array}{l}216 \\
(61.19 \%)\end{array}$ & 29 & $\begin{array}{l}49 \\
(14.33 \%)\end{array}$ & $\begin{array}{l}293 \\
(85.67 \%)\end{array}$ & 40 & $\begin{array}{l}33 \\
(9.62 \%)\end{array}$ & $\begin{array}{l}310 \\
(90.38 \%)\end{array}$ & 39 & $\begin{array}{l}54 \\
(16.07 \%)\end{array}$ & $\begin{array}{l}282 \\
(83.93 \%)\end{array}$ & 46 \\
\hline Upper back & $\begin{array}{l}120 \\
(34.09 \%)\end{array}$ & $\begin{array}{l}232 \\
(65.91 \%)\end{array}$ & 39 & $\begin{array}{l}42 \\
(12.24 \%)\end{array}$ & $\begin{array}{l}301 \\
(87.76 \%)\end{array}$ & 39 & $\begin{array}{l}26 \\
(7.56 \%)\end{array}$ & $\begin{array}{l}318 \\
(92.44 \%)\end{array}$ & 38 & $\begin{array}{l}46 \\
(13.69 \%)\end{array}$ & $\begin{array}{l}290 \\
(86.31 \%)\end{array}$ & 46 \\
\hline Elbows & $\begin{array}{l}42 \\
(11.97 \%)\end{array}$ & $\begin{array}{l}309 \\
(88.03 \%)\end{array}$ & 31 & $\begin{array}{l}27 \\
(7.89 \%)\end{array}$ & $\begin{array}{l}315 \\
(92.11 \%)\end{array}$ & 40 & $\begin{array}{l}14 \\
(4.12 \%)\end{array}$ & $\begin{array}{l}326 \\
(95.88 \%)\end{array}$ & 42 & $\begin{array}{l}25 \\
(7.46 \%)\end{array}$ & $\begin{array}{l}310 \\
(92.54 \%)\end{array}$ & 47 \\
\hline Wrists/hands & $\begin{array}{l}93 \\
(26.35 \%)\end{array}$ & $\begin{array}{l}260 \\
(73.65 \%)\end{array}$ & 29 & $\begin{array}{l}41 \\
(11.99 \%)\end{array}$ & $\begin{array}{l}301 \\
(88.01 \%)\end{array}$ & 40 & $\begin{array}{l}22 \\
(6.47 \%)\end{array}$ & $\begin{array}{l}318 \\
(93.53 \%)\end{array}$ & 42 & $\begin{array}{l}44 \\
(13.17 \%)\end{array}$ & $\begin{array}{l}290 \\
(86.83 \%)\end{array}$ & 48 \\
\hline Lower back & $\begin{array}{l}200 \\
(56.66 \%)\end{array}$ & $\begin{array}{l}153 \\
(43.34 \%)\end{array}$ & 29 & $\begin{array}{l}99 \\
(28.70 \%)\end{array}$ & $\begin{array}{l}246 \\
(71.30 \%)\end{array}$ & 31 & $\begin{array}{l}58 \\
(17.06 \%)\end{array}$ & $\begin{array}{l}282 \\
(82.94 \%)\end{array}$ & 42 & $\begin{array}{l}109 \\
(32.54 \%)\end{array}$ & $\begin{array}{l}226 \\
(67.46 \%)\end{array}$ & $4 \pi$ \\
\hline Hips/thighs & $\begin{array}{l}70 \\
(19.89 \%)\end{array}$ & $\begin{array}{l}282 \\
(80.11 \%)\end{array}$ & 30 & $\begin{array}{l}32 \\
(9.33 \%)\end{array}$ & $\begin{array}{l}311 \\
(90.67 \%)\end{array}$ & 39 & $\begin{array}{l}17 \\
(5.03 \%)\end{array}$ & $\begin{array}{l}321 \\
(94.97 \%)\end{array}$ & 44 & $\begin{array}{l}27 \\
(8.04 \%)\end{array}$ & $\begin{array}{l}309 \\
(91.96 \%)\end{array}$ & 46 \\
\hline Knees & $\begin{array}{l}90 \\
(25.57 \%)\end{array}$ & $\begin{array}{l}262 \\
(74.43 \%)\end{array}$ & 30 & $\begin{array}{l}34 \\
(9.94 \%)\end{array}$ & $\begin{array}{l}308 \\
(90.06 \%)\end{array}$ & 40 & $\begin{array}{l}24 \\
(7.06 \%)\end{array}$ & $\begin{array}{l}316 \\
(92.94 \%)\end{array}$ & 42 & $\begin{array}{l}38 \\
(11.34 \%)\end{array}$ & $\begin{array}{l}297 \\
(88.66 \%)\end{array}$ & 47 \\
\hline Ankles/feet & $\begin{array}{l}98 \\
(27.84 \%)\end{array}$ & $\begin{array}{l}254 \\
(72.16 \%)\end{array}$ & 30 & $\begin{array}{l}38 \\
(11.11 \%)\end{array}$ & $\begin{array}{l}304 \\
(88.89 \%)\end{array}$ & 40 & $\begin{array}{l}21 \\
(6.25 \%)\end{array}$ & $\begin{array}{l}315 \\
(93.75 \%)\end{array}$ & 46 & $\begin{array}{l}40 \\
(11.94 \%)\end{array}$ & $\begin{array}{l}295 \\
(88.06 \%)\end{array}$ & 47 \\
\hline
\end{tabular}

TABLE 5: Percentages of self-reported musculoskeletal symptoms among all participants ( $N=382$, $100 \%$ )

MS: musculoskeletal

*If the numbers do not add up to the total, it represents missing data, and the shown \% figure represents a valid percentage

\section{Discussion}

Work-related musculoskeletal diseases contribute massively to workplace absenteeism as they make employees avoid going to work [16]. These disorders can be more severe among healthcare workers as most of them are engaged in both non-physical and physical work. The objective of this study was to examine the prevalence of work-related MSCs among health practitioners in Al'Qassim, Saudi Arabia, and to identify different risk factors that contribute to the development of WMSDs. We wanted to establish results that can be used by health organizations to assess the problem and execute what is best for healthcare providers.

The total prevalence of WMSDs was found to be $54.7 \%$, which is much higher than in a study done in India, where they reported a prevalence of $26.4 \%$ [3]. However, it is a bit lower than what has been found among other studies (79.7\%) [13]. This variation can be due to external or internal factors affecting the studies' results, but the numbers in each study are considered of remarkable significance. The prevalence of MSCs was quite as expected with some variations pertaining to different demographics and work-related risk factors that were considered in other studies.

We found that female practitioners have a higher risk for WMSDs compared to their male counterparts (OR: 1.173; 95\% CI: 0.331-4.162), which is similar to a study that was conducted in Turkey and another one from Bangladesh $[5,17]$. If we look at the work experience and how it correlates with WMSDs, we can appreciate a remarkable variation between different studies; an Indian study concluded that there is no direct link between WMSDs in healthcare providers and their vast years of experience $(p=0.331)$ [3]. A similar observation was made in another study in Nigeria, which found no relationship between the years of experience and MSCs $(\mathrm{p}=0.873$ ) [18]. However, our study concluded otherwise and found that participants with more than six years of experience are at higher risk for developing WMSDs (OR: 2.342; 95\% CI: 1.062$5.168 ; \mathrm{p}=0.0350$ ). This difference between the results can be due to differences in the age of the 
participants or the greater responsibilities and duties given to senior healthcare providers in our region. Speaking of age, we found that being younger than 30 years makes people less vulnerable to WMSDs (OR: 0.74), and this is similar to what has been found in the Indian study (OR: 0.82); this can be linked with young people's ability to exercise more, as exercise also showed protective results (OR: 0.86 ). We also found that It is not the number of shifts that can result in WMSDs $(p=0.64)$, but the duration of the shifts $(p=0.013)$, and this matches with the findings of a similar study in Jeddah, Saudi Arabia $(p=0.01)[6]$. However, this finding differs from what was seen in another study conducted among Indian health practitioners $(\mathrm{p}=0.96)$ [3]. Such differences may be attributed to the difference in the nature of work during shifts in different regions and countries compared to Saudi Arabia.

Among the MSCs that were most frequently reported, low back pain (56.66\%) was the predominant complaint, followed by neck pain (46.05\%) and pain in the shoulders (38.81\%). Pain in the elbow was found to be the least reported complaint (11.97\%), and this almost matches with the order of complaints as reported by participants in other studies [3,6]. By observing these numbers and frequencies, we found that low back pain among all is the most frequent complaint that we need to pay special attention to; this MSC has been already studied separately and the results were almost similar to the one we found (70.09\%) [19]. This has urged us to propose that healthcare organizations should pay special attention to low back pain. Even though the pain in the lower back, neck, and shoulders was the most frequently reported MSC, few of the participants sought medical help for these complaints during the last 12 months.

Participants reported that most of the job risk factors were causing them trouble and often played a part in their developing WMSDs. Risk factors like performing the same task over and over again (37.96\%), working in awkward and cramped positions (20.29\%), and working in the same positions (standing, bending over, sitting, kneeling) for long periods (35.8\%) were frequently reported. Similar job risk factors were reported by other studies $[3,14]$. This should trigger the alarm for health education systems around the world to start making healthcare providers pay special attention to what they do at work and maintain healthy physical positioning at work.

This study has some limitations. Similar to some other studies, our study was limited by its cross-sectional design and failure of some participants to answer the questionnaire in full. We recommend conducting a larger, multicentric, prospective study that would include participants from different regions in Saudi Arabia. Such a study will contribute significantly to enhancing the accuracy of the results and understanding WMSDs better. This study and its results should encourage educational programs to develop programs on musculoskeletal disorder prevention strategies as it will reduce the rate of WMSDs and ensure the health and wellbeing of healthcare practitioners.

\section{Conclusions}

As per the findings of this study, we found the highest prevalence of WMSDs in physiotherapists based on the percentage of affected participants from a single specialty. However, nursing was found to be the most affected specialty based on the total number of participants from all specialties. Pain in the lower back, shoulders, and neck were the most frequently reported MSCs. Forceful work, forward bending of the trunk, and neck flexion of more than 20 degrees were found to be the most commonly self-reported ergonomic hazards at workstation relating to the development of WMSDs. We also observed that performing the same task over and over again was the major job risk factor among participants, followed by treating an excessive number of patients in one day.

\section{Appendices}

Questionnaire questions

Part One (demographic details)

Personal details:

What is your age?

What is your sex?

What is your height $(\mathrm{m})$ ?

What is your weight $(\mathrm{Kg})$ ?

What is your marital status?

Occupational and medical history:

What is your current occupation?

In which hospital do you work?

How many years of professional experience do you have? 


\section{Cureus}

How many numbers of shift(s) do you work?

What is the duration of 1 shift?

Have you suffered from musculoskeletal pain/discomfort in the past?

Arthritis?

Osteoporosis?

Disk Herniation?

Low back pain?

Are you suffering from musculoskeletal pain/discomfort now?

Lifestyle and habits:

Do you involve yourself regularly in sports/exercise/yoga?

Do you exercise regularly?

Are you a Smoker?

Are you an Alcoholic?

Part Two (self-reported ergonomic hazards at workstation):

Does your job involve neck flexion of more than 20 degrees?

Does your job involve arm level higher than shoulder?

Does your job involve repetitive work for more than 4 minutes?

Does your job involve forceful work?

Does your job involve the forward bending of the trunk?

Does your job involve lateral bending or twisting of the trunk?

Does your job involve prolonged sitting (more than 20 minutes)?

Does your job involve prolonged standing (more than 20 minutes)?

Does your job involve lifting, pulling, or pushing?

Part Three (job risk factor identification) (score of 0 to 10):

(A score of 0 to 1 indicates that the job factor poses "no problem," a score of 2 to 7 indicates that the job factor poses "minimal to moderate problem," and a score of 8 to 10 indicates that the job factor poses a "major problem")

Performing the same task over and over?

Treating an excessive number of patients in 1 day?

Performing manual orthopedic techniques (joint mobilizations, soft tissue mobilization)?

Not enough rest breaks or pauses during the workday?

Working in awkward and cramped positions?

Working in the same positions (standing, bending over, sitting, kneeling) for long periods?

Bending or twisting your back in an awkward way?

Working near or at your physical limits?

Reaching or working away from your body?

Continuing to work while injured or hurt?

Lifting or transferring dependent patients?

Working with confused or agitated patients?

Carrying, lifting, or moving heavy materials or equipment (e.g., continuous passive motion)?

Unanticipated sudden movement or fall by patients?

Assisting patients during gait activities? 


\section{Cureus}

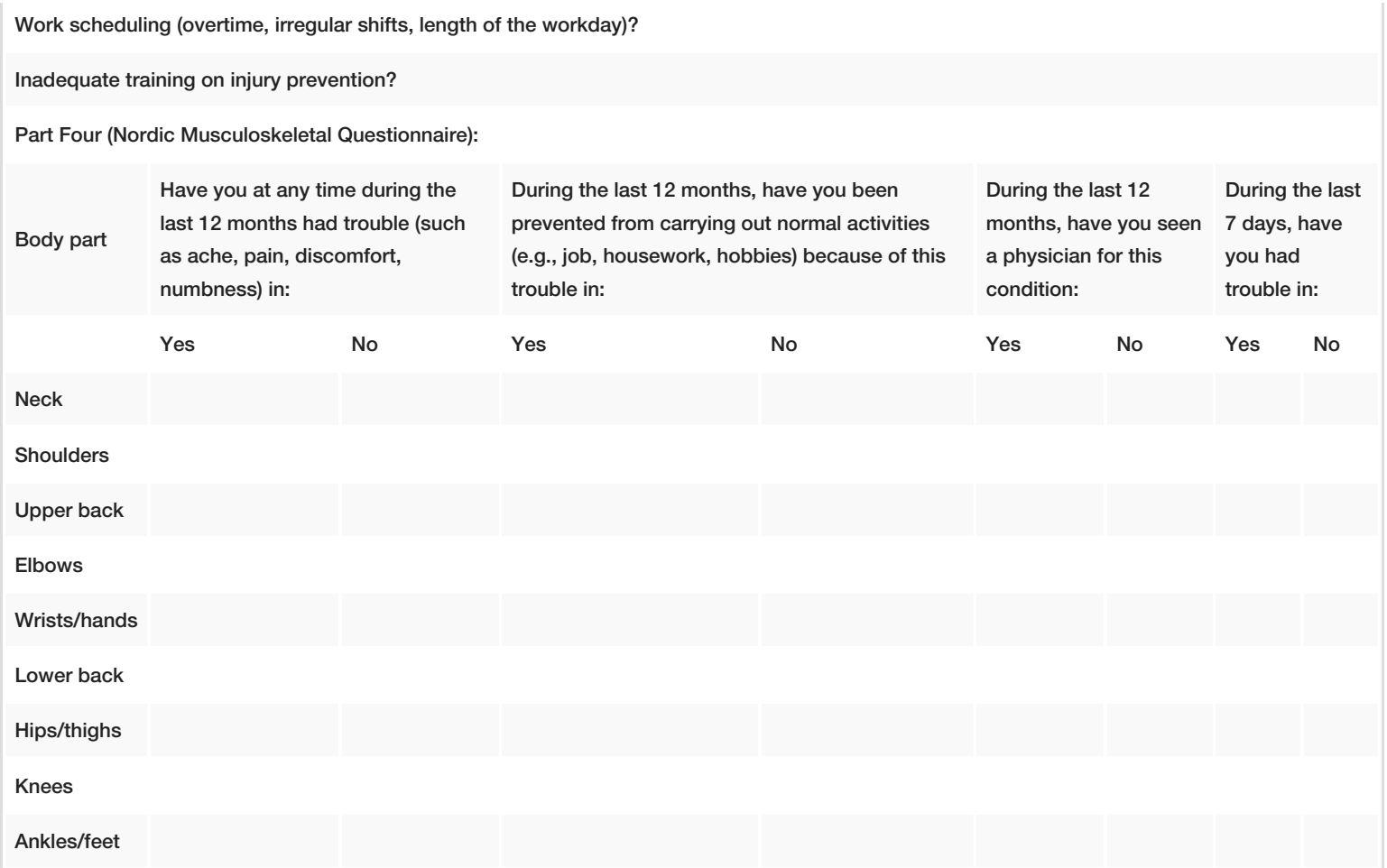

\section{TABLE 6: Work-related musculoskeletal disorders questionnaire}

Informed Consent for Research: Work-related Musculoskeletal Disorders Among Medical Practitioners at Hospitals of Al'Qassim Region, Saudi Arabia

Voluntary participation: participation in this study is voluntary. You will suffer no penalty nor loss of any benefits to which you are otherwise entitled should you decide not to participate. Withdrawal from this research study will not affect you in any way

Confidentiality: Your identity and medical record, as a participant in this research study, will remain confidential with respect to any publications of the results of this study

Authorization of voluntary participant who is not expected to obtain any direct benefit

A: I acknowledge that I have (read/or had explained to me) in a language I understand, the attached research participant Information sheet and that $\mathrm{Dr}$....................has explained to me the nature and purpose of this study. I have had the opportunity to ask any questions I had with respect to this study and all questions I asked were answered to my satisfaction

B: I understand the purpose of this study and I voluntarily accepted it after sufficient explanation

C: I understand that I am free to withdraw this authorization and discontinue participation in this study at any time. The consequences and risks, if any, of such withdrawal during the course of the study have been explained to me

D: I confirm that I have (read/or had read to me), the foregoing authorization and that all blanks or statements requiring completion were properly completed before I signed

I confirm that I have accurately (translated and/or read) the information to the subject: Signature:

Participant name: Hospital ID\#:

TABLE 7: Informed consent form given to participants

\section{Additional Information \\ Disclosures}

Human subjects: Consent was obtained by all participants in this study. National Bioethics Committee (NCBE) at Qassim province issued approval 1441-1641656. This study was approved by the National Bioethics Committee (NCBE) at Qassim province with the approval number 1441-1641656. Animal subjects: All authors have confirmed that this study did not involve animal subjects or tissue. Conflicts of interest: 
In compliance with the ICMJE uniform disclosure form, all authors declare the following: Payment/services info: All authors have declared that no financial support was received from any organization for the submitted work. Financial relationships: All authors have declared that they have no financial relationships at present or within the previous three years with any organizations that might have an interest in the submitted work. Other relationships: All authors have declared that there are no other relationships or activities that could appear to have influenced the submitted work.

\section{References}

1. Yelin EH, Felts WR: A summary of the impact of musculoskeletal conditions in the United States . Arthritis Rheum. 1990, 33:750-755. 10.1002/art.1780330520

2. Yelin EH, Henke CJ, Epstein WV: Work disability among persons with musculoskeletal conditions. Arthritis Rheum. 1986, 29:1322-1333. 10.1002/art.1780291104

3. Yasobant S, Rajkumar P: Work-related musculoskeletal disorders among health care professionals: a crosssectional assessment of risk factors in a tertiary hospital, India. Indian J Occup Environ Med. 2014, 18:75-81. 10.4103/0019-5278.146896

4. Punnett L, Wegman DH: Work-related musculoskeletal disorders: the epidemiologic evidence and the debate. J Electromyogr Kinesiol. 2004, 14:13-23. 10.1016/j.jelekin.2003.09.015

5. Koyuncu N, Karcioglu Ö: Musculoskeletal complaints in healthcare personnel in hospital: an interdepartmental, cross-sectional comparison. Medicine (Baltimore). 2018, 97:e12597. 10.1097/MD.0000000000012597

6. Attar SM: Frequency and risk factors of musculoskeletal pain in nurses at a tertiary centre in Jeddah, Saudi Arabia: a cross sectional study. BMC Res Notes. 2014, 7:61. 10.1186/1756-0500-7-61

7. Smith DR, Wei N, Ishitake T, Wang RS: Musculoskeletal disorders among Chinese medical students. Kurume Med J. 2005, 52:139-146. 10.2739/kurumemedj.52.139

8. Thornton LJ, Barr AE, Stuart-Buttle C, et al.: Perceived musculoskeletal symptoms among dental students in the clinic work environment. Ergonomics. 2008, 51:573-586. 10.1080/00140130701728277

9. Smith DR, Leggat PA: Musculoskeletal disorders among rural Australian nursing students. Aust J Rural Health. 2004, 12:241-245. 10.1111/j.1440-1854.2004.00620.x

10. Ando S, Ono Y, Shimaoka M, Hiruta S, Hattori Y, Hori F, Takeuchi Y: Associations of self estimated workloads with musculoskeletal symptoms among hospital nurses. Occup Environ Med. 2000, 57:211-216. 10.1136/oem.57.3.211

11. Alghadir A, Zafar H, Iqbal ZA: Work-related musculoskeletal disorders among dental professionals in Saudi Arabia. J Phys Ther Sci. 2015, 27:1107-1112. 10.1589/jpts.27.1107

12. Smith DR, Wei N, Zhao L, Wang RS: Musculoskeletal complaints and psychosocial risk factors among Chinese hospital nurses. Occup Med (Lond). 2004, 54:579-582. 10.1093/occmed/kqh117

13. Alexopoulos EC, Burdorf A, Kalokerinou A: A comparative analysis on musculoskeletal disorders between Greek and Dutch nursing personnel. Int Arch Occup Environ Health. 2006, 79:82-88. 10.1007/s00420-0050033-z

14. Tinubu BM, Mbada CE, Oyeyemi AL, Fabunmi AA: Work-related musculoskeletal disorders among nurses in Ibadan, South-west Nigeria: a cross-sectional survey. BMC Musculoskelet Disord. 2010, 11:12. 10.1186/1471-2474-11-12

15. Picavet HS, Schouten JS: Musculoskeletal pain in the Netherlands: prevalences, consequences and risk groups, the DMC(3)-study. Pain. 2003, 102:167-178. 10.1016/s0304-3959(02)00372-x

16. Wynne-Jones G, Cowen J, Jordan JL, Uthman O, Main CJ, Glozier N, van der Windt D: Absence from work and return to work in people with back pain: a systematic review and meta-analysis. Occup Environ Med. 2014, 71:448-456. 10.1136/oemed-2013-101571

17. Rahman M, Chowdhury A, Zaman MS, Sultana N, Amin MB, Hossain MM: Work-related musculoskeletal disorders among health care professionals. UpDCJ. 2017, 7:4-9. 10.3329/updcj.v7i1.33302

18. Akodu AK, Ashalejo ZO: Work-related musculoskeletal disorders and work ability among hospital nurses . J Taibah Univ Med Sci. 2019, 14:252-261. 10.1016/j.jtumed.2019.02.009

19. Çınar-Medeni Ö, Elbasan B, Duzgun I: Low back pain prevalence in healthcare professionals and identification of factors affecting low back pain. J Back Musculoskelet Rehabil. 2017, 30:451-459. 10.3233/BMR-160571 